\title{
Efficacy and Safety of Treatments to Improve Dyspareunia in Breast Cancer Survivors: A Systematic Review
}

\author{
Nicolás Mendoza $^{a}$ Rosalía Carrión ${ }^{a}$ Loreto Mendoza-Huertas ${ }^{a}$ \\ Ana Rosa Jurado ${ }^{a, b}$ \\ ${ }^{a}$ Departamento de Obstetricia y Ginecología, Universidad de Granada, Granada, Spain; ${ }^{b}$ Instituto Europeo de \\ Sexologia, Marbella, Spain
}

\section{Keywords \\ Dyspareunia · Female sexual dysfunction - Breast cancer . Breast cancer survivor - Vaginal therapy}

\begin{abstract}
Objective: To analyse all available evidence to validate the effectiveness of a local intervention in the treatment of dyspareunia in breast cancer survivors (BCS). Methods: We searched the Institute of Scientific Information Web of Knowledge, MEDLINE, PubMed, Scopus, and Cochrane databases for all articles published in peer-reviewed journals up to April 2019. The PICOS standards were: (population) BCS with dyspareunia; (intervention) any type of vulvovaginal treatment; (main outcome) frequency and severity of dyspareunia; (study design) clinical studies. Results: The literature search strategy identified 252 articles, of which 233 were excluded at various stages of the search. Finally, we systematically reviewed 19 studies, 8 with local hormonal therapies, 7 with local non-hormonal therapies, 3 with laser therapy, and 1 with other interventions. Of the studies, 7 were randomized control trials and 11 were prospective observations.
\end{abstract}

Most of the interventions were shown to be effective and safe in the improvement of dyspareunia. Conclusion: In addition to the traditional options already analysed in other current reviews, other interesting options are highlighted (such as laser or local dehydroepiandrosterone [DHEA]). Further work on dyspareunia should make use of high-quality trials with large numbers of samples to obtain evidence that could adequately demonstrate key methodological characteristics and harmful effects.

๑) 2020 S. Karger AG, Basel

\section{Introduction}

Breast cancer (BC) is the most common type of cancer (accounting for $28.7 \%$ of all cancers) and has the highest mortality rate $(15.5 \%)$ of all cancers affecting women [1]. However, the number of BC survivors (BCS) has also increased due to early detection programs and improved

The study was registered at www.propero.org (CRD42019127832). 


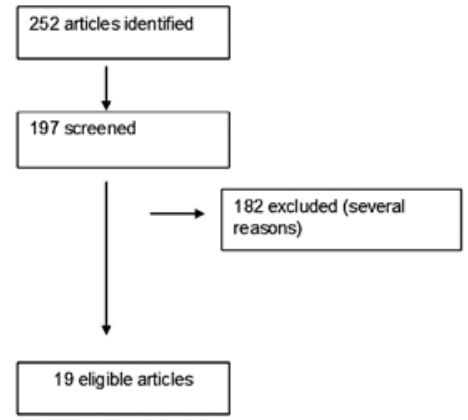

Fig. 1. Flow chart of the literature search.

treatments; therefore, $\mathrm{BC}$ has the highest survival rate among all cancers affecting women [2].

In BCS, sexual health is affected after treatment, having an impact on all phases of sexual response, with vaginal atrophy being particularly problematic. In this regard, dyspareunia is common in BCS, particularly in those women who are treated with aromatase inhibitors (AIs) [3].

In previously published articles on sexual health in BCS, we recommended that an adequate sexual health approach in BCS should be delivered through a multidisciplinary team, in order to minimize female sexual dysfunction (FSD) and relationship issues [4].

Overall, there are few randomized controlled trials (RCTs) evaluating treatments for dyspareunia in BCS women, and those that exist comprise small sample sizes and report mixed results. The most recommended option is the use of vaginal moisturizers and lubricants. Only after consultation with the patient's oncologist and comprehensive counselling about potential risks should local oestrogen treatment be used [4]. However, in recent years new therapeutic options have emerged for the treatment of postmenopausal vaginal symptoms that deserve to be evaluated in these women.

The purpose of this systematic review is to analyse all available evidence to validate the effectiveness of local interventions in the treatment of perimenopausal and postmenopausal BCS with dyspareunia.

\section{Methods}

\section{Selection of Studies}

We searched the Scientific Information Web of Knowledge (MEDLINE, PubMed, Scopus, and Cochrane databases) for all articles (in any language) published in peer-reviewed journals up to April 2019. The search criteria were applied to each database and combined with the available database-specific filters. Other publications were identified by manually searching through a reference list of papers highlighted by the search as well as key reviews. Press reports published in peer-reviewed journals and reports available online prior to publication were also considered.

The PICOS (Population, Intervention, Comparators, Outcomes, Study Design) criteria were developed a priori to guide the scope of the review, along with the procedures, selection, and synthesis of the literature search.

The selection criteria were as follows: (Population) patients with $\mathrm{BC}$ and dyspareunia; (Intervention) any type of pharmacological or local (vaginal) treatment; (Outcome) primary outcome: change in frequency, severity, and average mean scores of sexual symptoms measured with a validated instrument, secondary outcomes: quality of life; and (Study Design) clinical studies. Any complete article that met the inclusion criteria was reviewed in detail. Other related papers are for reference purposes only.

Exclusion criteria were other aetiologies of FSD, prevention (or treatment) of FSD, and the presence of other female cancers.

\section{Assessment of Study Quality and Data Synthesis}

We conducted a systematic review in accordance with PRISMA [5]. Qualitative and quantitative data were extracted from the papers included in the review using standardized data extraction tools from Jadal and CONSORT. The authors conducted an independent search, screening studies to include, extract, and examine data, and to synthesize the results. These authors independently evaluated the adequacy of the study design and the main methodological features in order to determine the validity of the study. The extracted data include the specific details about interventions, populations, research methods, and outcomes that are relevant to the reviewed questions and specific objectives. Disagreements were resolved through discussion and consensus.

\section{Data Extraction}

Two independent reviewers extracted the data from the included studies by using a specifically developed data extraction form based on the selection criteria. The extracted information includes a description of the study, participants, and findings based on the above outcomes. When there were no data (methods or results) of interest in the published paper, we contacted the author by email.

\section{Results}

As shown in Figure 1 (PRISMA flow chart), the literature search strategy identified 252 articles. Of these, 233 were excluded at different stages of the search. Finally, we systematically reviewed 19 studies, 8 of which were concerned with treatments based on local hormonal therapy [6-13], 7 with local non-hormonal therapy [14-20], 3 with laser [21-23], and 1 with other interventions [24].

Of the studies, 7 were RCTs $[12,13,15-19]$ and 11 were prospective observational studies [6-11, 14, 20, 2224]. Except for one [18] double-blind and random sequence generation, all RCTs were collected; allocation concealment was evident in only 2 RCTs $[13,17]$. One of the RCTs is a crossover study that includes the flushing phase [17]. Meta-analysis was not possible due to the heterogeneity and small sample size of the included studies.

A summary of the main features of the selected studies (study design, population, intervention, objective, and 


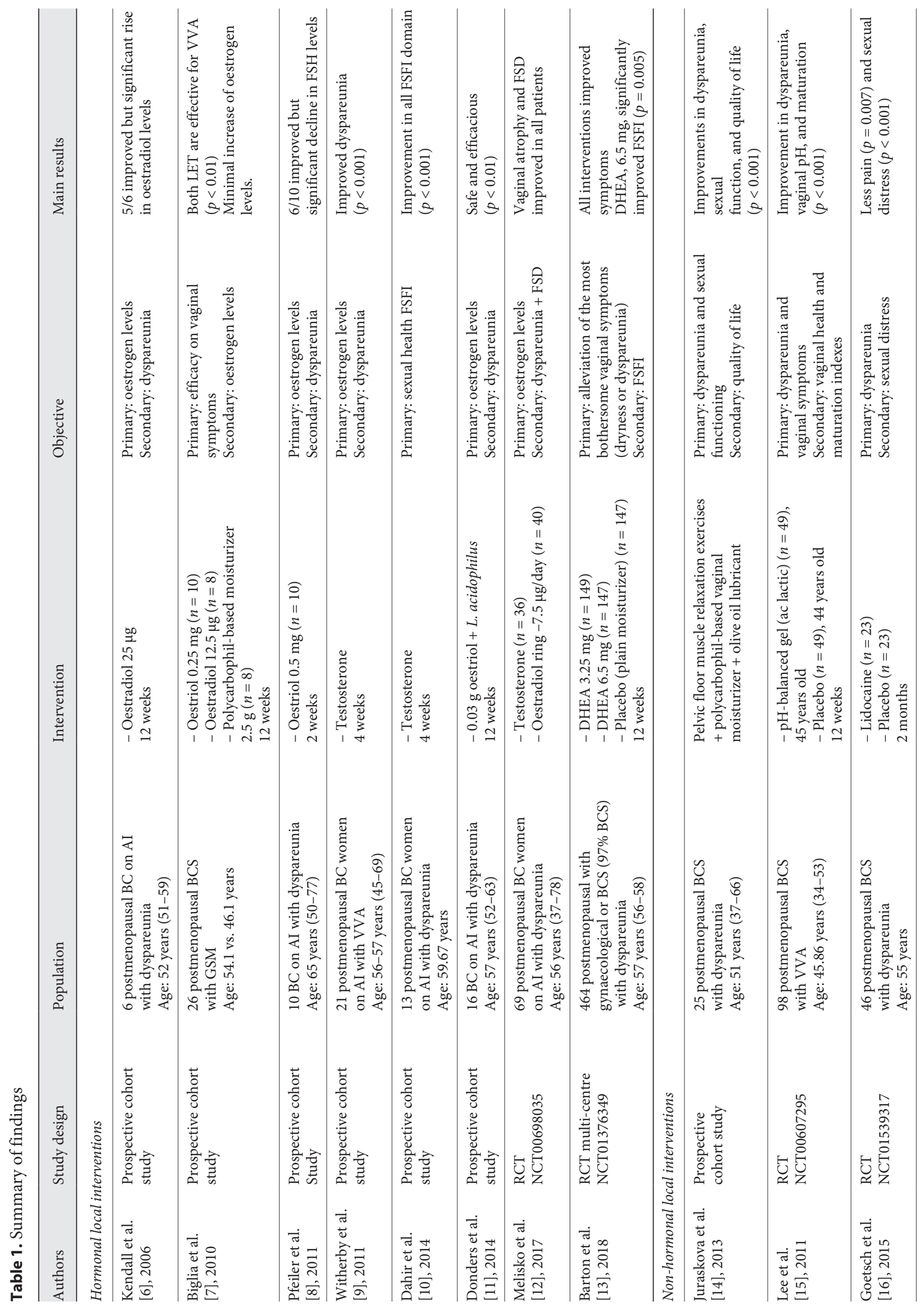




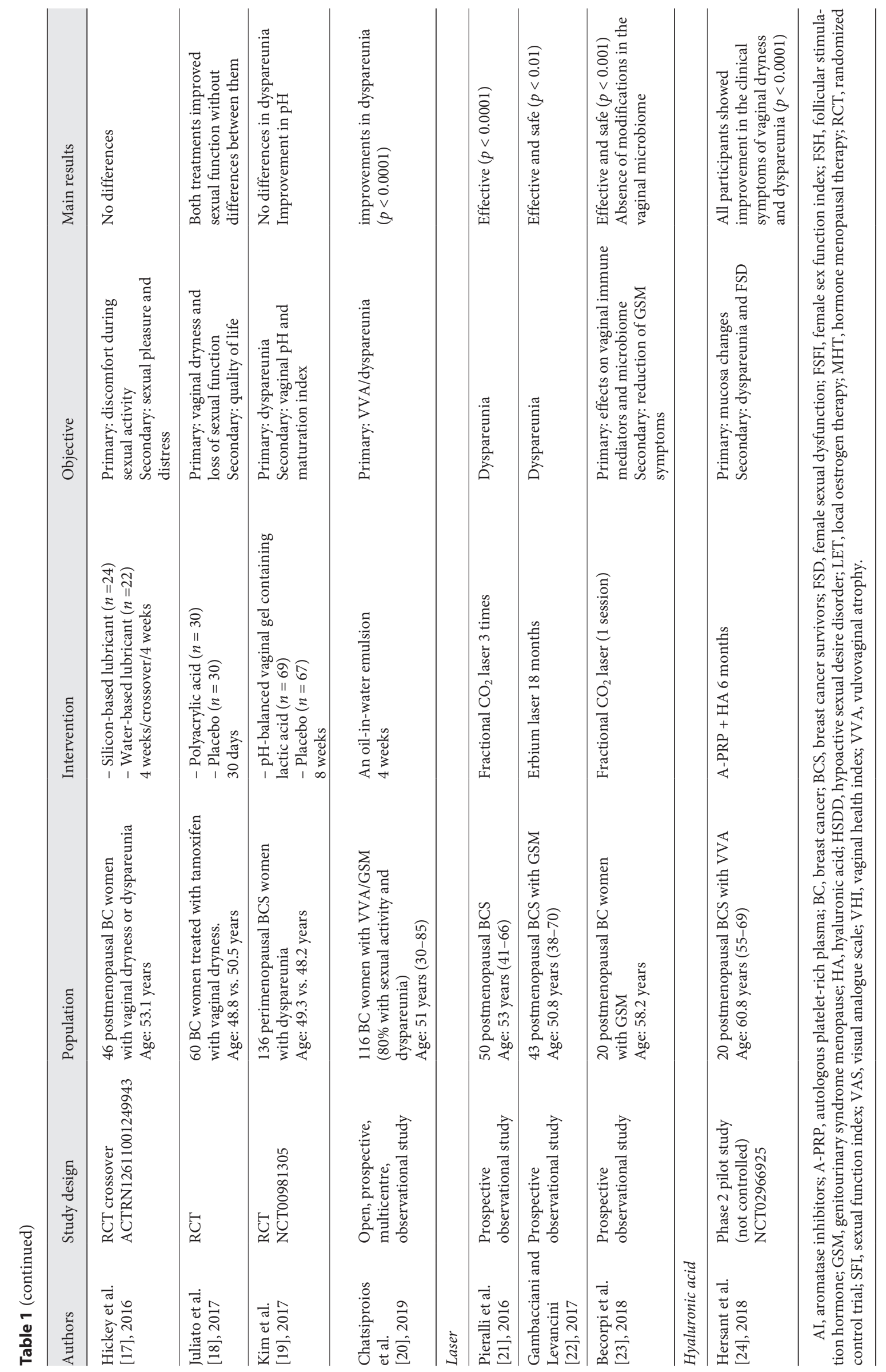


Table 2. Quality of studies included in the systematic review

\begin{tabular}{|c|c|c|c|c|c|c|c|}
\hline Authors & RCT & Random & Allocation & Blind & $\begin{array}{l}\text { Time } \\
\text { from BC }\end{array}$ & $\begin{array}{l}\text { Dropout, } \\
\%\end{array}$ & Adverse effects \\
\hline \multicolumn{8}{|l|}{ Hormonal local interventions } \\
\hline Kendall et al. [6], 2006 & - & - & - & - & - & None & Not described \\
\hline Biglia et al. [7], 2010 & - & - & - & - & - & 16 & Not described \\
\hline Pfeiler et al. [8], 2011 & - & - & - & - & - & None & - \\
\hline Witherby et al. [9], 2011 & - & - & - & - & + & 10 & + \\
\hline Dahir et al. [10], 2014 & - & - & - & - & + & 8 & Not described \\
\hline Donders et al. [11], 2014 & - & - & - & - & - & None & - \\
\hline Melisko et al. [12], 2016 & + & - & + & + & + & 10 & + \\
\hline Barton et al. [13], 2018 & + & - & + & + & - & 8 & + \\
\hline \multicolumn{8}{|c|}{ Non-hormonal local interventions } \\
\hline Juraskova et al. [14], 2013 & - & - & - & - & + & 36 & Not described \\
\hline Lee et al. [15], 2011 & + & + & - & + & - & 12 & + \\
\hline Goetsch et al. [16], 2015 & + & + & + & + & + & 11 & + \\
\hline Hickey et al. [17], 2016 & + & + & + & + & + & 8 & Not described \\
\hline Juliato et al. [18], 2017 & + & - & - & - & - & 13.3 & Not described \\
\hline Kim et al. [19], 2017 & + & + & - & + & - & 7.9 & + \\
\hline Chatsiproios et al. [20], 2019 & - & - & - & - & - & 8.5 & + \\
\hline \multicolumn{8}{|l|}{ Laser and hyaluronic acid } \\
\hline Pieralli [21], 2016 & - & - & - & - & - & None & + \\
\hline Gambacciani [22], 2017 & - & - & - & - & - & 14 & - \\
\hline Becorpi et al. [23], 2018 & - & - & - & - & - & None & Not described \\
\hline Hersant et al. [24], 2018 & - & - & - & - & - & None & - \\
\hline
\end{tabular}

main outcomes) is presented in Table 1. Although the mean age and age range of the patients were reported in all articles, BC diagnosis time was described in only 4 studies $[9,10,14,17]$. In all studies, the analysis of dyspareunia was an objective of the study, and in most of these (except for 2) this was the main objective. None of the studies had evaluated sexual behaviour prior to BC.

Most of the studies were considered to be of low to medium quality, primarily because they were not RCTs, or because of the small sample size, high dropout rates, or the fact that the adverse effects of the interventions were not described (see Table 2). All studies included heterosexual patients.

\section{Intervention Characteristics}

Hormonal therapy was used in 8 of the studies. In general, an improvement in dyspareunia was observed, but only 2 of these were RCTs [12,13]. In most of the studies on local oestrogen therapy, the main outcome was linked to oestrogen levels. Most of them were prospective cohort studies during 12 weeks with local preparations containing oestradiol $(7,7-25 \mu \mathrm{g}$ daily) $[6,7]$ or oestriol $(0.25-0.5 \mathrm{mg}$ daily) $[7,8]$. In 2 additional prospective cohort studies, testosterone vaginal cream (150-300 $\mu$ g daily) was used for 4 weeks $[9,10]$. One RCT used various doses of dehydroepiandrosterone (DHEA) daily for 12 weeks, demonstrating efficacy and safety in the treatment of dyspareunia in BCS women [13]. Most of them show that there was no significant difference in median serum oestradiol level before and after treatments, except in one study with vaginal oestradiol in BC patients under AI treatment [6]. No case of recurrence of $\mathrm{BC}$ was registered in any study with vaginal hormonal therapy [6-13] (for further details, see Table 1).

With regard to non-hormonal treatments, these involved the use of vaginal lubricants or moisturizers with a pH-balanced vaginal gel or similar. An improvement in dyspareunia was observed in $[14,15,18]$, and placebo also improved the dyspareunia in the study by Juliato et al. [18].

In 4 prospective cohort studies, local laser or autologous platelet-rich plasma plus hyaluronic acid was reported to improve dyspareunia. Apart from the pain that some women complained during vaginal probe insertion, no adverse event related to the procedures was recorder in any case [21-24].

\section{Discussion}

Although BC is an increasingly common disease, the number of survivors is also increasing due to early diagnosis and more effective treatment. Women who receive 
chemotherapy, particularly young women, are more likely to suffer from vaginal dryness, dyspareunia, decreased libido, and difficulty in reaching orgasm [25]. Among the ever-increasing health problems associated with BCS, women develop FSD, the most common and disturbing symptom of which is dyspareunia, which is often made worse by BC treatment, particularly AI. In most cases, hormonal therapy is not indicated or recommended in BCS women [26].

\section{Why Is It Important to Conduct This Systematic}

Review?

Trials that assess the effectiveness of dyspareunia treatment often exclude patients with other significant health problems, such as cancer. The diagnosis of FSD is becoming more frequent, and the need for effective and safe treatment is increasing. However, despite the growing importance of this topic, there is a lack of research and clarity of ideas. This systematic review updates the evidence included in a report published by the Spanish Menopause Society [4] and now only includes interventions used to treat FSD after BC.

\section{Strength}

This systematic review summarizes the state of the art regarding existing evidence on the effectiveness of local interventions for dyspareunia. Our review has identified 19 studies that recruited 990 patients with BC who had dyspareunia as the main symptom of FSD. The advantage of this evaluation lies in the systematic approach taken towards identifying and classifying existing evidence of BCS female dyspareunia and other FSD-specific interventions.

\section{Limitations}

Due to the heterogeneity of the research, it is fundamentally impossible to conduct a meta-analysis. Most studies consistently fail to specifically define degrees of dyspareunia; criteria for degrees of severity do not include dyspareunia scores or the ability of dyspareunia to disrupt sexual function patterns. In addition, women selfdiagnose and self-allocate with regard to the severity of the disorder (mild, moderate, or severe dyspareunia). The use of dryness as a diagnosis rather than a descriptor is also problematic.

Our systematic review studies differed in terms of design: some included a comparison of treatments without additional support for dyspareunia, whilst others compared interventions with active controls. Identifying the ideal care strategy for women with dyspareunia will be considered in the RCT in future studies by comparing the impact of new interventions on FSD.

Other important limitations to be noted are the small sample size of most studies, dropouts, and follow-up fail- ures, along with the beneficial effects of placebos in many settings. Most studies have a high risk of bias because they include samples of less than 50 participants in each trial. The trials differed not only in terms of the content of the intervention, but also with regard to the measurement of the results, which limited the combined analysis. In addition, the study lacked safety data. Generally, for most of the included studies, the quality of the evidence was medium to low.

BC patients could be diagnosed with dyspareunia for a variety of reasons: none of the studies indicated whether these were present in $\mathrm{BC}$ or whether the disease was present before or after treatment. In addition to dyspareunia, other emotional reasons, stress, and aging itself could be underlying causes of FSD. Therefore, not all FSDs are a direct consequence of $\mathrm{BC}$ or its treatment. In addition, interventions and recommendations for BCS without the disease are different from those for BC women, who may even be in treatment with drugs that impair sexual health, particularly AI.

\section{What Is Novel about Other Similar Reviews?}

Other systematic reviews have not found sufficient evidence to demonstrate the effectiveness of any type of intervention (pharmacological, mechanical, psychotherapeutic, or pelvic floor exercises). In addition, for medical treatment, the possible harmful effects have not been adequately recorded [27-30].

Our review was conducted in accordance with the Cochrane Library and we agreed to include only treatments for dyspareunia, rather than preventive measures [31]. But, unlike the Cochrane Library, we have not imposed restrictions on observational studies because these include interesting options for using alternatives such as lasers or hyaluronic acid, which have not been compared in RCTs.

One of the mistakes that often occurs when developing the question in a systematic review is the failure to include all of the patient's important outcomes (such as toxicity or adverse effects). In this systematic review, we included a study to assess the effectiveness of dyspareunia treatment. Participants were women with $\mathrm{BC}$ who had developed dyspareunia due to $\mathrm{BC}$ treatment or $\mathrm{BC}$ itself. We only focus on the evaluation of pharmacological or local interventions.

\section{About the Strategies}

Generally, relatively few RCTs evaluated the treatment of dyspareunia after BC, while those that existed used small sample sizes and reported mixed results. The results showed significant evidence for regular use of various vaginal interventions to improve dyspareunia and dryness, and in many cases, sexual satisfaction. 


\section{Vaginal Hormonal Therapies}

Local preparations containing hormones (oestradiol, oestriol, testosterone, DHEA) have been studied in an uncontrolled way, with few cases, and an insufficient time period. Moreover, few studies have analysed their safety. In fact, in most studies, the main observed outcomes were in terms of effects on oestrogen levels following the intervention. In general, these treatments provide some relief from dyspareunia and dryness, but even at low doses, a local hormonal intervention can cause systemic absorption. Unfortunately, there were no RCTs designed to assess $\mathrm{BC}$ recurrence with long-term hormonal intervention.

In general, vaginal hormonal therapy is available as a tablet or cream and is effective in treating genital atrophy. However, early systemic absorption is a safety issue for patients with $\mathrm{BC}$ when the tissue is still atrophic, and it is only recommended for women who are not responsive to non-hormonal therapy and women being treated with AIs; these therapies should be avoided or only used for a very short period of time [32].

The most interesting study in this group was one that used various doses of DHEA [13]. This study analysed a group of heterogeneous women with any type of gynaecological cancer and BC, $97 \%$ of whom had a history of $\mathrm{BC}$, and some of whom received tamoxifen or AI. However, this is a large-sample multi-centre RCT demonstrating the efficacy and safety of vaginal DHEA in the treatment of dyspareunia. DHEA has been proposed as a treatment for vulvovaginal atrophy due to its intracellular transformation in oestrogen and androgen. Clinical improvement in postmenopausal women was observed following vaginal DHEA administration [33].

Considering the safety of the vaginal hormonal therapies, no case of recurrence of $\mathrm{BC}$ was registered in any study. Safety was the primary objective in most of them, and this was shown, as well as the absence of side effects in all of them [9-13].

Regarding oestrogenic blood levels, most studies include this value as the primary $[6,8,9,11,12]$ or secondary objective [7]. Most of them show that there was no significant difference in median serum oestradiol level before and after treatments. However, in BC patients under AI treatment, inconsistent results are observed with vaginal oestradiol $[6,12]$. Despite the safety provided by these studies, baseline elevation in oestradiol in AI patients complicates this assessment, so further study is required to understand oestradiol variability in this setting.

\section{Vaginal Lubricants and Moisturizers}

In our previous paper, non-hormonal vaginal lubricants and moisturizers were the treatment of choice for women in $\mathrm{BC}$ because they improved dryness and vulvovaginal blood flow [4].

Although there is limited evidence in patients with BC, vaginal moisturizers and lubricants are effective in treating dyspareunia and vaginal dryness when used regularly (3-5 times a week). There are three types of vaginal moisturizers and lubricants: pectin-based, water-based, and gels containing polycarbophil.

\section{Other Therapies}

In women with dyspareunia or vaginal dryness, other alternative therapies are increasingly being studied, particularly in cases where it is not possible to use other hormonal options. This is the case with local laser treatment, which is a good choice for BCS. In the three prospective observational studies of this review, which globally analysed 113 BCS women, the efficacy and safety of laser therapy were observed even when applied only once. Along with the use of DHEA, laser is one of the non-hormonal options that we highlight in this review. Unfortunately, we do not have enough RCTs to determine their effectiveness and safety, although some trials are already in progress.

\section{Future Research}

Nonetheless, our systematic review has identified some important areas for future research, particularly in laser-related areas, and several RCTs are underway. We expect that the results will contribute towards the development of an effective and safe alternative to treating dyspareunia. Larger double-blind studies should be conducted over a longer follow-up period to assess different treatment strategies for dyspareunia, and future research should also address the effectiveness and safety of dyspareunia interventions in BCS. In addition, there should be an extension of the intervention in order to monitor compliance for more than 3 months; this is important because the increase in major symptoms occurs between 1 and 3 months and relapses occur after discontinuation of use, similar to data in the general population [34].

\section{Conclusions}

Since improvements in both the diagnosis and treatment of BC mean that more women will become BCS, a significant proportion may have dyspareunia. In addition to the traditional options that have been analysed in other reviews, other interesting options have emerged (such as laser or local DHEA). High-quality trials with large numbers of samples are required to provide further evidence of dyspareunia or other dyspareunia treatments, particularly with regard to adequately identifying key methodological characteristics and harmful effects. 


\section{Acknowledgements}

This article was translated and edited by Oak-Fortress Proofreading International.

\section{Disclosure Statement}

The authors declare no conflicts of interest.

\section{Funding Sources}

There was no funding source and no editorial assistance for this systematic review.

\section{Author Contributions}

N. Mendoza and R. Carrión: conception and design of the study. N. Mendoza, R. Carrión, L. Mendoza-Huertas, and A.R. Jurado: data interpretation and preparation of the manuscript. All authors approved the final version of the manuscript.

\section{References}

1 De Angelis R, Sant M, Coleman MP, Francisci S, Baili P, Pierannunzio D, et al.; EUROCARE-5 Working Group. Cancer survival in Europe 1999-2007 by country and age: results of EUROCARE-5-a population-based study. Lancet Oncol. 2014 Jan; 15(1):23-34.

2 Hashim D, Boffetta P, La Vecchia C, Rota M, Bertuccio P, Malvezzi M, et al. The global decrease in cancer mortality: trends and disparities. Ann Oncol. 2016 May;27(5):926-33.

3 Sánchez-Borrego R, Mendoza N, Beltrán E, Comino R, Allué J, Castelo-Branco C, et al Position of the Spanish Menopause Society regarding the management of menopausal symptoms in breast cancer patients. Maturitas. 2013 Jul;75(3):294-300.

4 Mendoza N, Molero F, Criado F, Cornellana MJ, González E; Sexuality in Breast Cancer Survivors Group. Sexual health after breast cancer: recommendations from the Spanish Menopause Society, Federación Española de Sociedades de Sexología Sociedad Española de Médicos de Atención Primaria and Sociedad Española de Oncología Médica. Maturitas. 2017 Nov;105: 126-31.

5 Available from: http://www.prisma-statement.org/statement.htm.

6 Kendall A, Dowsett M, Folkerd E, Smith I. Caution: vaginal estradiol appears to be contraindicated in postmenopausal women on adjuvant aromatase inhibitors. Ann Oncol. 2006 Apr;17(4):584-7.

7 Biglia N, Peano E, Sgandurra P, Moggio G, Panuccio E, Migliardi M, et al. Low-dose vaginal estrogens or vaginal moisturizer in breast cancer survivors with urogenital atrophy: a preliminary study. Gynecol Endocrinol. 2010 Jun;26(6):404-12.

8 Pfeiler G, Glatz C, Königsberg R, Geisendorfer T, Fink-Retter A, Kubista E, et al. Vaginal estriol to overcome side-effects of aromatase inhibitors in breast cancer patients. Climacteric. 2011 Jun;14(3):339-44.

9 Witherby S, Johnson J, Demers L, Mount S, Littenberg B, Maclean CD, et al. Topical testosterone for breast cancer patients with vaginal atrophy related to aromatase inhibitors: a phase I/II study. Oncologist. 2011;16(4):42431.

10 Dahir M, Travers-Gustafson D. Breast cancer, aromatase inhibitor therapy, and sexual func- tioning: a pilot study of the effects of vaginal testosterone therapy. Sex Med. 2014 Apr;2(1): 8-15.

11 Donders G, Neven P, Moegele M, Lintermans A, Bellen G, Prasauskas V, et al. Ultra-lowdose estriol and Lactobacillus acidophilus vaginal tablets (Gynoflor $\left({ }^{\circledR}\right)$ ) for vaginal atrophy in postmenopausal breast cancer patients on aromatase inhibitors: pharmacokinetic, safety, and efficacy phase I clinical study. Breast Cancer Res Treat. 2014 Jun;145(2): 371-9.

12 Melisko ME, Goldman ME, Hwang J, De Luca A, Fang S, Esserman LJ, et al. Vaginal testosterone cream vs estradiol vaginal ring for vaginal dryness or decreased libido in women receiving aromatase inhibitors for early-stage breast cancer: a randomized clinical trial. JAMA Oncol. 2017 Mar;3(3):313-9.

13 Barton DL, Sloan JA, Shuster LT, Gill P, Griffin P, Flynn K, et al. Evaluating the efficacy of vaginal dehydroepiandosterone for vaginal symptoms in postmenopausal cancer survivors: NCCTG N10C1 (Alliance). Support Care Cancer. 2018 Feb;26(2):643-50.

14 Juraskova I, Jarvis S, Mok K, Peate M, Meiser B, Cheah BC, et al. The acceptability, feasibility, and efficacy (phase I/II study) of the OVERcome (Olive Oil, Vaginal Exercise, and MoisturizeR) intervention to improve dyspareunia and alleviate sexual problems in women with breast cancer. J Sex Med. 2013 Oct; 10(10):2549-58.

15 Lee YK, Chung HH, Kim JW, Park NH, Song YS, Kang SB. Vaginal pH-balanced gel for the control of atrophic vaginitis among breast cancer survivors: a randomized controlled trial. Obstet Gynecol. 2011 Apr;117(4):922-7.

16 Goetsch MF, Lim JY, Caughey AB. A Practical Solution for Dyspareunia in Breast Cancer Survivors: A Randomized Controlled Trial. J Clin Oncol. 2015 Oct;33(30):3394-400.

17 Hickey M, Marino JL, Braat S, Wong S. A randomized, double-blind, crossover trial comparing a silicone- versus water-based lubricant for sexual discomfort after breast cancer. Breast Cancer Res Treat. 2016 Jul;158(1):7990

18 Juliato PT, Rodrigues AT, Stahlschmidt R, Juliato CR, Mazzola PG. Can polyacrylic acid treat sexual dysfunction in women with breast cancer receiving tamoxifen? Climacteric. 2017 Feb;20(1):62-6.
19 Kim YH, Park S, Lee M, Hahn S, Jeon MJ. Effect of a pH-Balanced Vaginal Gel on Dyspareunia and Sexual Function in Breast Cancer Survivors Who Were Premenopausal at Diagnosis: A Randomized Controlled Trial. Obstet Gynecol. 2017 May;129(5):870-6.

20 Chatsiproios D, Schmidts-Winkler IM, König L, Masur C, Abels C. Topical treatment of vaginal dryness with a non-hormonal cream in women undergoing breast cancer treatment - An open prospective multicenter study. PLoS One. 2019 Jan;14(1):e0210967.

21 Pieralli A, Fallani MG, Becorpi A, Bianchi C, Corioni S, Longinotti M, et al. Fractional CO2 laser for vulvovaginal atrophy (VVA) dyspareunia relief in breast cancer survivors. Arch Gynecol Obstet. 2016 Oct;294(4):841-6.

22 Gambacciani M, Levancini M. Vaginal erbium laser as second-generation thermotherapy for the genitourinary syndrome of menopause: a pilot study in breast cancer survivors. Menopause. 2017 Mar;24(3):316-9.

23 Becorpi A, Campisciano G, Zanotta N, Tredici Z, Guaschino S, Petraglia F, et al. Fractional CO2 laser for genitourinary syndrome of menopause in breast cancer survivors: clinical, immunological, and microbiological aspects. Lasers Med Sci. 2018 Jul;33(5):104754.

24 Hersant B. SidAhmed-Mezi M, Belkacemi Y, Darmon F, Bastuji-Garin S, Werkoff G, Bosc R, Niddam J, Hermeziu O, La Padula S, Meningaud JP. Efficacy of injecting platelet concentrate combined with hyaluronic acid for the treatment of vulvovaginal atrophy in postmenopausal women with a history of breast cancer: a phase 2 pilot study. Menopause. 2018 Oct;25(10):1124-30.

25 Candy B, Jones L, Vickerstaff V, Tookman A King M. Interventions for sexual dysfunction following treatments for cancer in women. Cochrane Database Syst Rev. 2016 Feb; 2:CD005540.

26 Chang YC, Chang SR, Chiu SC. Sexual problems of patients with breast cancer after treatment: a systematic review. Cancer Nurs. 2019 Sep/Oct;42(5):418-25.

27 Maiorino MI, Chiodini P, Bellastella G, Giugliano D, Esposito K. Sexual dysfunction in women with cancer: a systematic review with meta-analysis of studies using the Female Sexual Function Index. Endocrine. 2016 Nov; 54(2):329-41. 
28 Seav SM, Dominick SA, Stepanyuk B, Gorman JR, Chingos DT, Ehren JL, et al. Management of sexual dysfunction in breast cancer survivors: a systematic review. Womens Midlife Health. 2015 Nov;1(1):9.

29 Bachmann GA, Ramachandra S. Management of sexual dysfunction in breast cancer survivors: a systematic review editorial--looking back to move ahead. Womens Midlife Health. 2015 Nov;1(1):8.

30 Mazzarello S, Hutton B, Ibrahim MF, Jacobs C, Shorr R, Smith S, et al. Management of urogenital atrophy in breast cancer patients: a systematic review of available evidence from randomized trials. Breast Cancer Res Treat. 2015 Jul;152(1):1-8.
31 Jing L, Zhang C, Li W, Jin F, Wang A. Incidence and severity of sexual dysfunction among women with breast cancer: a metaanalysis based on female sexual function index. Support Care Cancer. 2019 Apr;27(4): 1171-80

32 Farrell R; American College of Obstetricians and Gynecologists' Committee on Gynecologic Practice. ACOG Committee Opinion No. 659: The Use of Vaginal Estrogen in Women With a History of Estrogen-Dependent Breast Cancer. Obstet Gynecol. 2016 Mar;127(3):e93-6.
33 Labrie F, Archer DF, Koltun W, Vachon A, Young D, Frenette L, et al.; VVA Prasterone Research Group. Efficacy of intravaginal dehydroepiandrosterone (DHEA) on moderate to severe dyspareunia and vaginal dryness, symptoms of vulvovaginal atrophy, and of the genitourinary syndrome of menopause. Menopause. 2016 Mar;23(3):243-56.

34 Kenemans P, Bundred NJ, Foidart JM, Kubista E, von Schoultz B, Sismondi P, et al.; LIBERATE Study Group. Safety and efficacy of tibolone in breast-cancer patients with vasomotor symptoms: a double-blind, randomised, non-inferiority trial. Lancet Oncol. 2009 Feb;10(2):135-46 